\title{
Recorded infection control messages delay inter-professional communication but are not associated with COVID-19 prevalence or mortality: insights from a national switchboard analysis
}

\author{
Authors: Edd Maclean, ${ }^{A *}$ Rahul Ghelani, ${ }^{B *}$ Myra Adra, ${ }^{\mathrm{C}}$ Anshu Arora, ${ }^{C}$ Prasheena Naran, ${ }^{C}$ Heather Illing, ${ }^{C}$ \\ Megan Knight,, Sophie Banerjee, ${ }^{\mathrm{C}}$ Sam Myers, ${ }^{\mathrm{C}}$ Joanna Brecher, ${ }^{\mathrm{C}}$ Sarah Anderson, ${ }^{\mathrm{C}}$ Charlotte Aylward, ${ }^{\mathrm{C}}$ \\ Harmit Bindra, ${ }^{\mathrm{C}}$ Christine Carter, ${ }^{\mathrm{C}}$ Naim Dib, ${ }^{\mathrm{C}}$ Laura Ganis, ${ }^{\mathrm{C}}$ David Saliu, ${ }^{\mathrm{C}}$ Andrew Towler-Tinlin, ${ }^{\mathrm{C}}$ \\ Doaa Rajab Kerwat, ${ }^{C}$ Eleanor Richards, ${ }^{C}$ James Noble Johnston ${ }^{\mathrm{C}}$ and Barney Low ${ }^{\mathrm{C}}$
}

\begin{abstract}
Appropriate dissemination of information to the general public is a key component of the pandemic response. In 2018, recorded infection control advice messages were affixed to $30 \%$ of England's automated hospital switchboards during the seasonal influenza and norovirus outbreaks. As the majority of messages were mandatory for all callers, healthcare professionals using the hospital switchboard - including during time-critical emergencies - had their enquiries significantly delayed by these measures. Importantly, published analyses did not demonstrate an association between these messages and patient outcomes. As of May $2020,85 \%$ of NHS trusts made use of infection control messages; on average, these delayed healthcare professionals by 59.4 seconds per call, but had no clear association with patient outcomes from COVID-19. An ongoing national switchboard quality improvement project seeks to establish a gold standard whereby healthcare professionals with urgent enquiries can press ' $X$ ' to skip past infection control messages and have their calls triaged immediately.
\end{abstract}

KEYWORDS: COVID-19, infection control messages, switchboard response times, quality improvement

DOI: $10.7861 /$ clinmed.2020-0438

\section{Background}

The authors recently published an analysis of England's NHS hospital switchboard response times. ${ }^{1}$ Across England from 2018-2019, we assessed the average time taken for an external caller to contact a bleep operator capable of providing urgent assistance. Significantly, 115 hospitals (66\%) made use of automated switchboards; on average, these introduced an additional delay of 40 seconds per call versus human operators.

Authors: Aspecialist registrar in cardiology, William Harvey Research Institute, Queen Mary University of London, London, UK and Barts Health NHS Trust, London, UK; ${ }^{B}$ senior house officer, Chelsea and

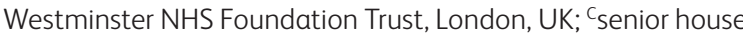
officer, Barts Health NHS Trust, London, UK; *joint first authors
In addition, $30 \%$ of automated switchboards provided recorded infection control advice; these messages further exacerbated the delays in communication, and population density-adjusted analyses of sentinel hospitals did not demonstrate an association between the use of infection control messages and local influenza admissions.

\section{Objectives}

These initial findings suggested that mandatory infection control messages significantly delayed contact with the target clinician without clearly improving patient outcomes, and hence we contacted all included NHS hospitals and suggested that they change their switchboards to provide healthcare professionals with the option for more urgent call triage. While the initial uptake of our best practice recommendations was encouraging, we subsequently repeated our data collection during the COVID-19 pandemic.

\section{Methods}

In May 2020, we contacted 212 switchboards across 162 hospital trusts in England. The presence and content of any recorded switchboard messages were documented. The switchboard response time was defined as the number of seconds before a human operator capable of contacting urgent assistance responded. No clinician calls or bleeps were connected.

Results

During the COVID-19 pandemic, the prevalence of infection control messages among automated switchboards has increased from $29.6 \%$ to $85.1 \%$. Accordingly, the national switchboard response time has deteriorated from $55.0 \pm 46$ to $64.5 \pm 46$ seconds $(p=0.013)$. Hospital switchboards with infection control messages responded an average of 60 seconds slower than those without (Table 1). Variations in COVID-19 prevalence and outcomes including the 22,639 in-hospital COVID-19 deaths in England as of 8 May 2020 - were assessed according to switchboard design, geographical location and population density (data taken from https://coronavirus.data.gov.uk and www.ons.gov.uk on 8 May 2020).

Community prevalence of COVID-19 per 100,000 population was not significantly different between hospitals using infection 
Table 1. Regional comparisons of hospital switchboard design, performance, and relationship with COVID-19 prevalence and mortality

\begin{tabular}{|c|c|c|c|c|c|c|}
\hline $\begin{array}{l}\text { Region } \\
\text { (number of } \\
\text { switchboards) }\end{array}$ & Switchboard design & $\begin{array}{l}\text { Number } \\
\text { (\% of } \\
\text { total) }\end{array}$ & $\begin{array}{l}\text { Time to } \\
\text { contact } \\
\text { bleep } \\
\text { operator } \\
\text { (seconds) }\end{array}$ & $\begin{array}{l}\text { Population } \\
\text { density* } \\
(\text { people/km²) }\end{array}$ & $\begin{array}{l}\text { Community prevalence } \\
\text { of COVID- } 19 \text { (cases } \\
\text { per } 100,000 \text { regional } \\
\text { population }{ }^{+} \text {) }\end{array}$ & $\begin{array}{l}\text { Hospital mortality } \\
\text { rate (deaths per } \\
1,000,000 \text { regional } \\
\text { population }{ }^{+} \text {) }\end{array}$ \\
\hline \multirow[t]{3}{*}{$\begin{array}{l}\text { England } \\
(n=212)\end{array}$} & $\begin{array}{l}\text { Hospital switchboards } \\
\text { with infection control } \\
\text { message (automated) }\end{array}$ & $\begin{array}{l}138 \\
(65.1 \%)\end{array}$ & $85.3 \pm 36.3$ & 432 & $235.6 \pm 54.8$ & $22.1 \pm 15.1$ \\
\hline & $\begin{array}{l}\text { Hospital switchboards } \\
\text { without infection } \\
\text { control message } \\
\text { (automated or human) }\end{array}$ & $\begin{array}{l}74 \\
(34.9 \%)\end{array}$ & $25.9 \pm 35.9$ & & $249.4 \pm 58.8$ & $20.0 \pm 12.9$ \\
\hline & p & & $<0.001$ & & 0.14 & 0.3 \\
\hline \multirow[t]{3}{*}{$\begin{array}{l}\text { East of } \\
\text { England } \\
(n=25)\end{array}$} & $\begin{array}{l}\text { Hospital switchboards } \\
\text { with infection control } \\
\text { message (automated) }\end{array}$ & $\begin{array}{l}19 \\
(76 \%)\end{array}$ & $91.9 \pm 36.7$ & 326 & 194.7 & $23.5 \pm 13.5$ \\
\hline & $\begin{array}{l}\text { Hospital switchboards } \\
\text { without infection } \\
\text { control message } \\
\text { (automated or human) }\end{array}$ & $\begin{array}{l}6 \\
(24 \%)\end{array}$ & $24.5 \pm 22.2$ & & & $29.6 \pm 10.6$ \\
\hline & p & & $<0.001$ & & & 0.33 \\
\hline \multirow[t]{3}{*}{$\begin{array}{l}\text { London } \\
(n=38)\end{array}$} & $\begin{array}{l}\text { Hospital switchboards } \\
\text { with infection control } \\
\text { message (automated) }\end{array}$ & $\begin{array}{l}29 \\
(76.3 \%)\end{array}$ & $104 \pm 32.6$ & 5701 & 288.9 & $34.1 \pm 20.2$ \\
\hline & $\begin{array}{l}\text { Hospital switchboards } \\
\text { without infection } \\
\text { control message } \\
\text { (automated or human) }\end{array}$ & $\begin{array}{l}9 \\
(23.7 \%)\end{array}$ & $25.6 \pm 34.2$ & & & $28.8 \pm 18.3$ \\
\hline & p & & $<0.001$ & & & 0.49 \\
\hline \multirow[t]{3}{*}{$\begin{array}{l}\text { Midlands } \\
(n=32)\end{array}$} & $\begin{array}{l}\text { Hospital switchboards } \\
\text { with infection control } \\
\text { message (automated) }\end{array}$ & $\begin{array}{l}21 \\
(65.6 \%)\end{array}$ & $84.2 \pm 36.2$ & 376 & 203.4 & $19.9 \pm 15.8$ \\
\hline & $\begin{array}{l}\text { Hospital switchboards } \\
\text { without infection } \\
\text { control message } \\
\text { (automated or human) }\end{array}$ & $\begin{array}{l}11 \\
(34.4 \%)\end{array}$ & $38.5 \pm 52.4$ & & & $18 \pm 9.4$ \\
\hline & p & & 0.008 & & & 0.72 \\
\hline \multirow[t]{3}{*}{$\begin{array}{l}\text { North East } \\
\text { and Yorkshire } \\
(n=35)\end{array}$} & $\begin{array}{l}\text { Hospital switchboards } \\
\text { with infection control } \\
\text { message (automated) }\end{array}$ & $\begin{array}{l}16 \\
(45.7 \%)\end{array}$ & $63.9 \pm 34.1$ & 341 & 277.9 & $17.1 \pm 6.6$ \\
\hline & $\begin{array}{l}\text { Hospital switchboards } \\
\text { without infection } \\
\text { control message } \\
\text { (automated or human) }\end{array}$ & $\begin{array}{l}19 \\
(54.3 \%)\end{array}$ & $21.3 \pm 23.4$ & & & $20.1 \pm 10$ \\
\hline & $\mathrm{p}$ & & $<0.001$ & & & 0.31 \\
\hline
\end{tabular}


Table 1. Regional comparisons of hospital switchboard design, performance, and relationship with COVID-19 prevalence and mortality (Continued)

\begin{tabular}{|c|c|c|c|c|c|c|}
\hline $\begin{array}{l}\text { Region } \\
\text { (number of } \\
\text { switchboards) }\end{array}$ & Switchboard design & $\begin{array}{l}\text { Number } \\
\text { (\% of } \\
\text { total) }\end{array}$ & $\begin{array}{l}\text { Time to } \\
\text { contact } \\
\text { bleep } \\
\text { operator } \\
\text { (seconds) }\end{array}$ & $\begin{array}{l}\text { Population } \\
\text { density* } \\
(\text { people/km²) }\end{array}$ & $\begin{array}{l}\text { Community prevalence } \\
\text { of COVID- } 19 \text { (cases } \\
\text { per } 100,000 \text { regional } \\
\text { population }^{+} \text {) }\end{array}$ & $\begin{array}{l}\text { Hospital mortality } \\
\text { rate (deaths per } \\
1,000,000 \text { regional } \\
\text { population }^{+} \text {) }\end{array}$ \\
\hline \multirow[t]{3}{*}{$\begin{array}{l}\text { North West } \\
(n=33)\end{array}$} & $\begin{array}{l}\text { Hospital switchboards } \\
\text { with infection control } \\
\text { message (automated) }\end{array}$ & $\begin{array}{l}19 \\
(57.6 \%)\end{array}$ & $77.1 \pm 39.1$ & 520 & 303.7 & $26.4 \pm 10.9$ \\
\hline & $\begin{array}{l}\text { Hospital switchboards } \\
\text { without infection } \\
\text { control message } \\
\text { (automated or human) }\end{array}$ & $\begin{array}{l}14 \\
(42.4 \%)\end{array}$ & $33.3 \pm 48$ & & & $16.6 \pm 14.9$ \\
\hline & $\mathrm{p}$ & & 0.008 & & & 0.037 \\
\hline \multirow[t]{3}{*}{$\begin{array}{l}\text { South East } \\
(n=27)\end{array}$} & $\begin{array}{l}\text { Hospital switchboards } \\
\text { with infection control } \\
\text { message (automated) }\end{array}$ & $\begin{array}{l}24 \\
(88.9 \%)\end{array}$ & $77.9 \pm 38$ & 481 & 209.3 & $16.5 \pm 7.2$ \\
\hline & $\begin{array}{l}\text { Hospital switchboards } \\
\text { without infection } \\
\text { control message } \\
\text { (automated or human) }\end{array}$ & $\begin{array}{l}3 \\
(11.1 \%)\end{array}$ & $28.5 \pm 33.3$ & & & $17.8 \pm 2.8$ \\
\hline & $p$ & & 0.04 & & & 0.76 \\
\hline \multirow[t]{3}{*}{$\begin{array}{l}\text { South West } \\
(n=22)\end{array}$} & $\begin{array}{l}\text { Hospital switchboards } \\
\text { with infection control } \\
\text { message (automated) }\end{array}$ & $\begin{array}{l}12 \\
(54.5 \%)\end{array}$ & $91.6 \pm 29.9$ & 236 & 124.5 & $10.7 \pm 8.7$ \\
\hline & $\begin{array}{l}\text { Hospital switchboards } \\
\text { without infection } \\
\text { control message } \\
\text { (automated or human) }\end{array}$ & $\begin{array}{l}10 \\
(45.5 \%)\end{array}$ & $37 \pm 35.7$ & & & $10.6 \pm 8.5$ \\
\hline & p & & $<0.001$ & & & 0.97 \\
\hline
\end{tabular}

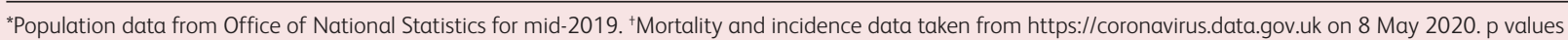
as per two-tailed unpaired $t$ tests.

control messages or not $(235.6 \pm 54.8$ cases versus $249.4 \pm 58.8$ cases, $p=0.14)$. Use of a recorded infection control message was not associated with a reduction in per hospital COVID-19 mortality rate per million population ( $22.1 \pm 15.1$ deaths versus $20.0 \pm 12.9$ deaths, $p=0.3$ ), and regional prevalence ( $\%$ ) of infection control messages did not correlate with reduced mortality (Pearson's $\mathrm{R}^{2}$ $0.074, p=0.56$ ).

\section{Discussion}

We suggest that, for clinicians in need of urgent telephone assistance from another hospital, infection control messages affixed to automated switchboards significantly delay help, are superfluous information for medical professionals and, while they may be informative for the general public, are not assciated with a clear mortality benefit. As such, we continue to call for the institution of a national standard for the hospital switchboard, in which healthcare professionals with urgent clinical queries can press ' $X$ ' to bypass any recorded messages and have their calls triaged immediately. Prior to the COVID-19 outbreak, the response to these recommendations had been positive with considerable uptake nationwide and early evidence of improved inter-hospital communication times. Concerns that the general public might exploit a switchboard redesign to triage non-clinical calls were mitigated by the use of key phrases in the revised recorded messages; for example, a trust in the South East demonstrated that 'Welcome to ' $X$ ' Trust. If you are a healthcare professional in need of urgent assistance, press 1 to access the emergency bleep system. For all other callers, please hold' was not prone to misuse. Further quality improvement measures to encourage these changes, and repeat data collection to assess their efficacy, will be performed in late 2020 .

\section{Reference}

1 Ghelani R, Maclean E, Adra M et al. Identifying avoidable switchboard delays in England's NHS hospitals: phase one of the national SWITCH project. Acute Med 2019;18:208-13.

Address for correspondence: Dr Edd Maclean, 3rd floor, 1 St Martin's Le Grand, London EC1A 4AS, UK. Email: e.maclean@nhs.net 